\title{
Prévision de la fissuration par fatigue en présence de contraintes résiduelles
}

\author{
Bertrand Journeta ${ }^{\mathrm{a}}$ et FABrice Congourdeau \\ EADS CCR, 12 rue Pasteur, BP 76, 92152 Suresnes, France
}

Reçu le 2 juillet 2004, accepté le 5 novembre 2004

\begin{abstract}
Résumé - La démonstration de la sécurité des structures aéronautiques repose sur des calculs de scénarii de fissuration en fatigue. Les calculs de propagation doivent prendre en compte la présence éventuelle de contraintes résiduelles qui sont induites par les procédés d'assemblage des pièces. Les alésages des jonctions de fuselage sont concernés par cet aspect. Une approche prévisionnelle de la fissuration en fatigue des alésages expansés et montés en interférence est présentée. L'approche est validée sur des essais représentatifs des cas de service. Une application de l'approche à des calculs d'aide à la conception est présentée.
\end{abstract}

Mots clés : Alésage / expansion / interférence / contraintes résiduelles / fissuration en fatigue / prévision / mécanique de la rupture

\begin{abstract}
Fatigue crack growth prediction in presence of residual stresses. The integrity of aeronautical structures must be proved on the basis of fatigue cracking scenarios. The crack growth calculations must include the presence of would-be residual stresses which are induced by the assembling processes. Fastener holes of fuselage joints are typical sites with built-up residual stress fields. An approach to predict the fatigue crack growth at cold expanded and interference fit holes is presented. Fatigue tests have been run on in-service representative cases to validate the approach. An application of the approach to the design of fastener holes is presented.
\end{abstract}

Key words: Hole / cold expansion / interference fitting / residual stress / fatigue crack growth / prediction / fracture mechanics

\section{Introduction}

Les structures aéronautiques sont dimensionnées en fatigue de façon à ce qu'aucun endommagement n'apparaisse durant leur exploitation. En ce qui concerne les structures métalliques, l'endommagement par fissuration est une préoccupation majeure. Les causes potentielles d'amorçage des fissures sont multiples : la fatigue, la corrosion, les défauts de fabrication. La réglementation aérienne demande de démontrer que si un tel dommage se produisait, alors qu'il ne devrait pas (cas hypothétique et pessimiste) il ne mettrait pas en péril la sécurité de l'appareil. Les zones travaillantes jugées critiques, vis-à-vis de tels scénarii hypothétiques, sont donc dimensionnées en tolérance aux dommages. Cela veut dire que les aéronefs peuvent voler en toute sécurité en présence de fissures, le tout est qu'elles n'atteignent pas une certaine taille critique. À cet effet, le dossier de justification donne la fréquence des inspections à effectuer de façon à détecter

\footnotetext{
a Auteur correspondant : bertrand.journet@eads.net
}

à temps les fissures éventuelles. Un des éléments clés de la démonstration est le calcul de propagation des fissures en fatigue. Le résultat du calcul doit être acceptable, c'est-àdire conservatif, du côté de la sécurité, et assez proche de la réalité pour ne pas pénaliser la masse de la structure ni les programmes d'inspection.

Les calculs de propagation sont effectués dans le cadre de la mécanique linéaire élastique de la rupture. Il s'agit de calculer les facteurs d'intensité de contrainte en pointe de fissure $K$ et d'utiliser les lois de fissuration des matériaux. Certaines zones critiques, comme les alésages des jonctions de fuselage, sont plus difficiles à calculer du fait de la présence de contraintes résiduelles induites par le procédé de montage des fixations. Les procédés d'expansion des alésages et de montage en interférence des rivets de fixation dans les alésages sont bénéfiques en fatigue car les temps de propagation des fissures sont rallongés. Le procédé d'expansion consiste à faire passer un brunissoir au travers de l'alésage. La partie centrale de cet outil, en forme d'olive avec un diamètre supérieure 


\section{Nomenclature}

\begin{tabular}{|c|c|}
\hline$a$ & longueur d'une fissure en coin d'alésage, côté intérieur de l'alésage, mm \\
\hline$a / c$ & rapport de forme d'une fissure coin de forme quart d'ellipse \\
\hline$B$ & épaisseur d'éprouvette, mm \\
\hline$c$ & longueur d'une fissure en surface d'une plaque, $\mathrm{mm}$ \\
\hline$C_{\mathrm{p}}$ & coefficient de retard \\
\hline $\mathrm{d} a / \mathrm{d} N$ & vitesse de fissuration, mm.cycle ${ }^{-1}$ \\
\hline $\mathrm{d} c / \mathrm{d} N$ & vitesse de fissuration, mm.cycle ${ }^{-1}$ \\
\hline$(\mathrm{d} c / \mathrm{d} N)_{w, 2 \mathrm{D}}$ & vitesse de fissuration, en présence de contraintes résiduelles, fissure traversante, mm.cycle ${ }^{-1}$ \\
\hline$(\mathrm{d} c / \mathrm{d} N)_{w, 3 \mathrm{D}}$ & vitesse de fissuration, en présence de contraintes résiduelles, fissure de coin, mm.cycle ${ }^{-1}$ \\
\hline$D$ & diamètre d'alésage, mm \\
\hline $\mathrm{EF}$ & éléments finis \\
\hline$H$ & hauteur d'éprouvette, mm \\
\hline$K$ & facteur d'intensité de contrainte, $\mathrm{MPa} \sqrt{m}$ \\
\hline$K_{\mathrm{op}}$ & facteur d'intensité de contrainte d'ouverture, $\mathrm{MPa} \sqrt{m}$ \\
\hline$K_{\max }$ & valeur maximale du facteur d'intensité de contrainte, $\mathrm{MPa} \sqrt{m}$ \\
\hline$K_{\min }$ & valeur minimale du facteur d'intensité de contrainte, $\mathrm{MPa} \sqrt{m}$ \\
\hline$K_{\text {res }}$ & facteur d'intensité de contrainte résiduel, $\mathrm{MPa} \sqrt{m}$ \\
\hline$K_{\mathrm{ref}, 2 \mathrm{D}}$ & facteur d'intensité de contrainte du cas de référence, fissure traversante, $\mathrm{MPa} \sqrt{m}$ \\
\hline$K_{\text {ref,3D }}$ & facteur d'intensité de contrainte du cas de référence, fissure de coin, $\mathrm{MPa} \sqrt{m}$ \\
\hline$K_{w, 2 \mathrm{D}}$ & facteur d'intensité de contrainte, avec contraintes résiduelles, fissure traversante, $\mathrm{MPa} \sqrt{m}$ \\
\hline$K_{w, 3 \mathrm{D}}$ & facteur d'intensité de contrainte, avec contraintes résiduelles, fissure de coin, $\mathrm{MPa} \sqrt{m}$ \\
\hline$\Delta K_{\text {eff }}$ & variation du facteur d'intensité de contrainte efficace, $\mathrm{MPa} \sqrt{m}, \Delta K_{\mathrm{eff}}=K_{\mathrm{max}}-K_{\mathrm{op}}$ \\
\hline$\Delta K_{\text {eff }, \text { ref }, 2 \mathrm{D}}$ & variation du facteur d'intensité de contrainte efficace, cas de référence, fissure traversante, $\mathrm{MPa} \sqrt{m}$ \\
\hline$\Delta K_{\text {eff }, \text { ref }, 3 \mathrm{D}}$ & variation du facteur d'intensité de contrainte efficace, cas de référence, fissure de coin, $M P a \sqrt{m}$ \\
\hline$\Delta K_{\mathrm{eff}, w, 2 \mathrm{D}}$ & variation de $K$ efficace, avec contraintes résiduelles, fissure traversante, $\mathrm{MPa} \sqrt{m}$ \\
\hline$\Delta K_{\mathrm{eff}, w, 3 \mathrm{D}}$ & variation de $K$ efficace, avec contraintes résiduelles, fissure de coin, $\mathrm{MPa} \sqrt{m}$ \\
\hline$N$ & nombre de cycles de fatigue \\
\hline$S_{22}$ & contrainte perpendiculaire au plan de fissuration, $\mathrm{MPa}$ \\
\hline$S_{\max }$ & valeur maximale de la contrainte de fatigue, $\mathrm{MPa}$ \\
\hline$S_{\min }$ & valeur minimale de la contrainte de fatigue, $\mathrm{MPa}$ \\
\hline$\sigma$ & contrainte, $\mathrm{MPa}$ \\
\hline$\sigma_{22}$ & contrainte perpendiculaire au plan de fissuration, $\mathrm{MPa}$ \\
\hline$r$ & distance polaire d'un point du solide à la pointe de la fissure, mm \\
\hline$r_{\mathrm{p}}$ & rayon de la zone plastique en pointe de fissure, $\mathrm{mm}$ \\
\hline$R$ & rapport du chargement de fatigue, $R=S_{\min } / S_{\max }$ \\
\hline$R_{\mathrm{s}}$ & rapport $R$ stabilisé \\
\hline$W$ & largeur d'éprouvette, mm \\
\hline
\end{tabular}

à celui de l'alésage, écrouit ce dernier et y laisse des contraintes résiduelles de compression après son passage. Le montage en interférence consiste à installer un rivet, dans l'alésage, en le « frappant » de façon à forcer un flot de matière visant à le gonfler et ainsi écrouir l'alésage qui l'entoure et qui le pincera par retour élastique. Ces procédés sont utilisés séparément ou en combinaison. Ce sont ces contraintes résiduelles d'écrouissage et de pincement qui augmentent les durées de vie en fatigue. Ces effets sont pris en compte chez AIRBUS par des coefficients issus d'essais qui sont appliqués aux lois de fissuration dans les calculs. Ces coefficients demandent à être optimisés car ils sont trop conservatifs. Cela nécessite de pouvoir calculer les facteurs $K$ en prenant en compte les contraintes résiduelles.

Les approches disponibles dans la littérature reposent essentiellement sur des principes de superposition élastique appliqués dans le cadre de la mécanique linéaire élastique de la rupture. Un type d'approche consiste à calculer un facteur $K$ « résiduel $» K_{\text {res }}$, qu'il suffirait d'ajouter au facteur $K$ dû à la sollicitation de service. Le facteur $K$ résiduel peut être calculé à partir de la distribution locale des contraintes résiduelles et de fonctions de poids [1]. Un autre type d'approche consiste à calculer 
une contrainte effective locale, résultant d'une superposition élastique des contraintes locales résiduelles et de la contrainte locale mécanique due à la sollicitation de service, et de l'injecter dans une formule classique de facteur $K[2]$.

Les procédés d'expansion et d'interférence induisent des contraintes en bord d'alésage (circonférentielles ou perpendiculaires à la fissure) qui sont respectivement négatives et positives. Ces approches prévoiraient donc respectivement un ralentissement et une accélération de la fissure. Or les essais montrent que, pour chacun des procédés, la fissure en bord d'alésage est retardée [3].

À la demande d'AIRBUS France, EADS CCR, l'un des deux centres communs de recherche du groupe EADS situé à Suresnes, a donc décidé de revoir la mécanique de la rupture des alésages fissurés en présence de contraintes résiduelles. La démarche proposée s'appuie sur des calculs par éléments finis en élastoplasticité pour analyser le champ des contraintes en pointe de fissure. Cette approche, qui simule les procédés d'expansion et d'interférence, permet de gérer la superposition des contraintes résiduelles avec celles de service et de rendre compte de tout effet plastique au voisinage de l'alésage susceptible d'exercer un effet sur la fissuration.

Afin de répondre à un besoin d'AIRBUS France concernant l'amélioration des outils de calculs en tolérance aux dommages, EADS CCR a développé une approche permettant de prendre en compte les points évoqués. L'objet de cet article est de présenter une synthèse des travaux menés par EADS CCR sur ce thème, selon le plan suivant :

- présentation de la méthode;

- vérification de la méthode sur des cas de référence dans lesquels l'alésage est brut d'usinage, sans contraintes résiduelles significatives;

- validation de la méthode sur des cas d'interférence ou d'expansion représentatifs d'alésages de jonctions de panneaux de fuselage, dans lesquels l'alésage est habité par des contraintes résiduelles résultant du procédé de montage $[4,5]$;

- application de la méthode à des calculs prospectifs pour aide à la conception d'alésages expansés montés en interférence (combinaison expansion + interférence) $[5,6]$.

\section{Présentation de la méthode}

La méthode présentée se décompose en plusieurs étapes :

- Étape 1 : simulation du procédé d'expansion ou d'interférence pour la génération des contraintes résiduelles.

- Étape 2 : superposition de l'effort de service au résultat précédant pour l'analyse du champ de contrainte en pointe de fissure et la détermination du facteur $K$.
- Étape 3a : prévision de la vitesse de fissuration pour une fissure traversante, à partir de la connaissance du facteur $K$.

- Étape 3b : prévision de la vitesse de fissuration pour une fissure coin en forme de quart d'ellipse, à partir de la connaissance du facteur $K$.

Les données d'entrée sont les paramètres du procédé, la loi de comportement statique et les lois de fissuration du matériau. La méthode calcule le facteur d'intensité de contrainte et réalise les prévisions en appliquant des lois de fissuration.

La méthode repose sur des calculs élastoplastiques par éléments finis et sont réalisés sur le code ABAQUS. Les quatre étapes de calcul sont décrites ci-dessous et sont résumées en figure 1. Le cœur de l'approche est la simulation du procédé pour générer le champ de contraintes résiduelles dans un modèle fissuré puis la détermination du facteur $K$ dans la zone élastique en avant de la fissure.

\section{Étape 1 : simulation du procédé}

- Expansion : Un pré-traitement concernant spécifiquement l'expansion est conduit. Un modèle axisymétrique $2 \mathrm{D}^{1 / 2}$ permet de simuler le procédé (passage de l'outil dans l'alésage) et d'obtenir dans toute l'épaisseur de la plaque le profil de contrainte tangentielle résiduelle induite par le procédé. Les contraintes résiduelles d'expansion ne sont pas homogènes dans l'épaisseur. Seul le profil en surface (face d'entrée du brunissoir) est retenu pour l'analyse car il correspond au lieu d'amorçage des fissures. Pour diminuer l'ampleur des calculs des étapes suivantes, on recommence la simulation de l'expansion en 2D, par dilatation mécanique du diamètre, en contraintes planes, pour reproduire le champ de contrainte en surface obtenu en $2 \mathrm{D}^{1 / 2}$. Cela permettra ensuite de conduire une analyse en mécanique de la rupture en $2 \mathrm{D}$.

- Interférence : simulation 2D par éléments finis en élasto-plasticité, en contraintes planes, du procédé d'interférence, avec mise en place du contact entre un rivet infiniment rigide et l'alésage. Le contact est sans frottement. La simulation consiste à dilater mécaniquement le diamètre du rivet.

$\rightarrow$ Données d'entrée : loi de comportement élastoplastique du matériau avec écrouissage cinématique, diamètre du rivet, taux d'interférence, diamètre de l'alésage, taux d'expansion, géométrie de l'éprouvette. Les taux sont calculés à partir des diamètres initiaux et finaux de l'alésage.

\section{Étape 2}

Calcul du facteur $K$ en pointe d'une fissure traversante soumise à la fois aux contraintes résiduelles et aux contraintes mécaniques de vol et noté $K_{w, 2 \mathrm{D}}$.

- Création d'une fissure traversante dans le maillage précédent, de longueur $a$. Les tailles d'éléments les plus fins sont de $20 \mu \mathrm{m}$, dans le voisinage de la pointe de la fissure. 


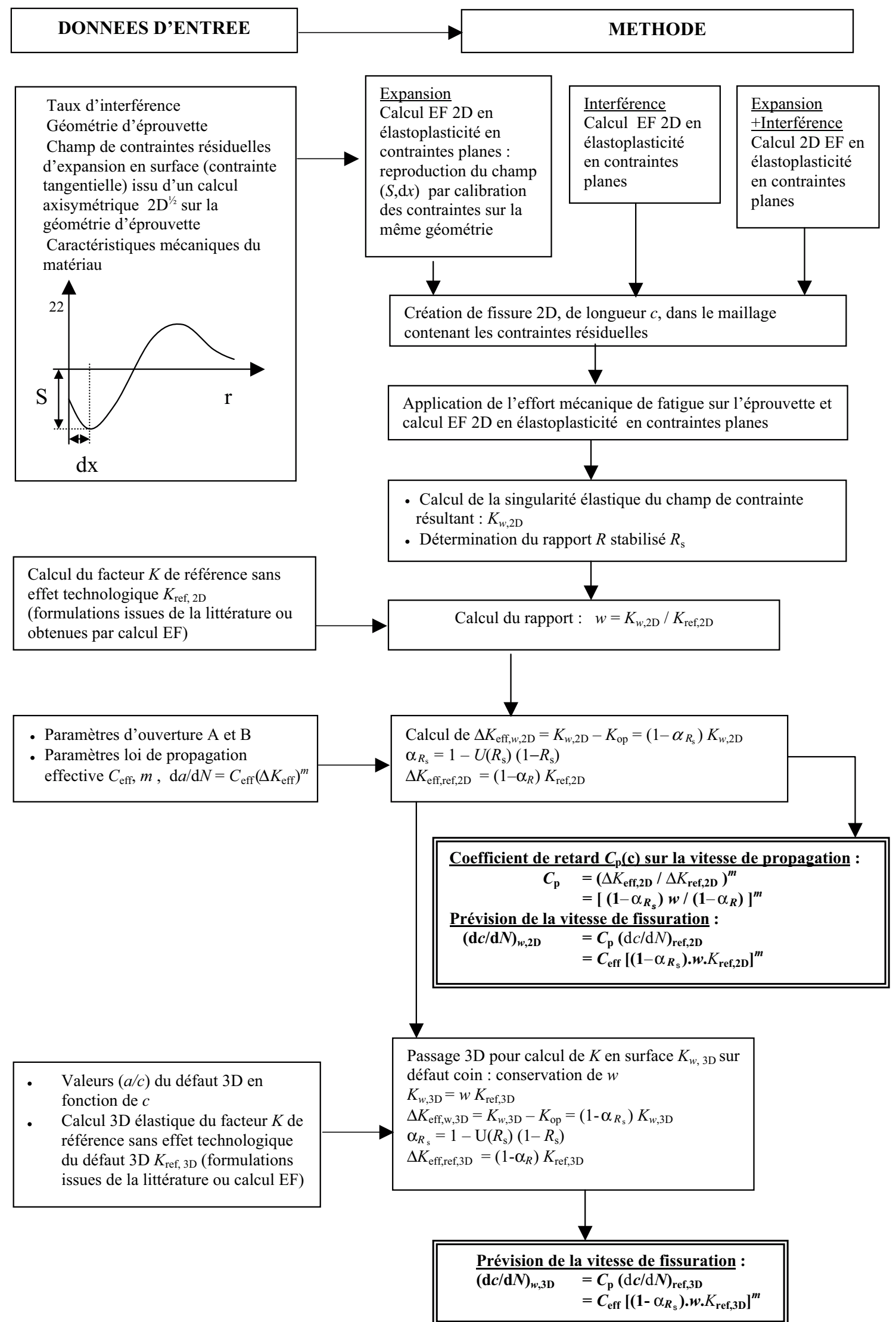

Fig. 1. Description de la méthode pour l'expansion et/ou l'interférence. 


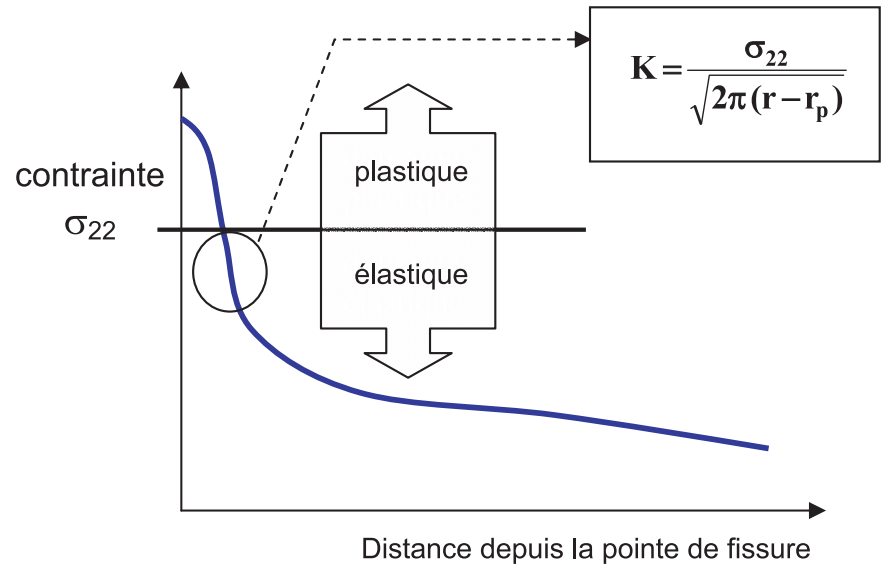

Fig. 2. Principe de détermination de la singularité élastique à partir de champ de contraintes en pointe de fissure obtenue par calcul éléments finis en élastoplasticité (étape 2 de la méthode).

- Application de la contrainte maximale de vol $S_{\max }$, à l'aide d'une simulation 2D par éléments finis en élasto-plasticité, en contraintes planes, au maillage fissuré précédent pour obtenir, en pointe de fissure, le champ des contraintes $(\sigma)$ résultant des contraintes résiduelles et de vol.

- Analyse du champ $(\sigma)$ pour déterminer le facteur d'intensité de contrainte élastique $K_{w, 2 \mathrm{D}}$, en avant de la zone plastique en pointe de fissure, en opérant un changement de coordonnées par la correction d'Irwin : $K_{w, 2 \mathrm{D}}=\sigma \sqrt{2 \pi\left(r-r_{\mathrm{p}}\right)}$, le rayon de la zone plastique $r_{\mathrm{p}}$ étant calculé par exploitation des calculs EF. $K_{w, 2 \mathrm{D}}$ est pris comme la moyenne des trois premiers points de calculs dans la zone élastique (Fig. 2).

- Détermination du rapport $R$ vu par la fissure. En effet la présence de contraintes résiduelles va changer le rapport $R$ de chargement que va voir la fissure. Le rapport $R$ intervient dans la fissuration. On appellera $R_{\mathrm{s}}$ ce rapport $R$, s pour « stabilisé $»$.

- Calcul de $w=K_{w, 2 \mathrm{D}} / K_{\text {ref,2D }}$, où $K_{\text {ref,2D }}$ est le facteur $K$ d'une fissure traversante soumise seulement à la contrainte de vol et qui peut être calculé de manière classique.

$\rightarrow$ Données d'entrée : loi de comportement élastoplastique du matériau avec écrouissage cinématique, longueur de fissure.

\section{Étape 3a}

Prévision de la fissuration, fissure traversante, soumise à la fois aux contraintes résiduelles et aux contraintes mécaniques de vol.

- Calcul de $\Delta K_{\text {eff }, w, 2 \mathrm{D}}=K_{w, 2 \mathrm{D}}-K_{\text {op }}$, en utilisant la relation d'Elber, adaptée éventuellement aux petites fissures, et le rapport $R_{\mathrm{s}}$.

- Application de la loi de fissuration du matériau de base (coefficients $C_{\text {eff }}$ et exposant $m$ ) pour prédire la vitesse avec l'effet de retard dû au procédé : $(\mathrm{d} a / \mathrm{d} N)_{w}=C_{\mathrm{eff}}\left(\Delta K_{\mathrm{eff}, w, 2 \mathrm{D}}\right)^{m}$.
- Établissement d'abaques de retard, donnant un coefficient de retard $C_{\mathrm{p}}$, sur la vitesse de fissuration, en fonction de la longueur de fissure $c$ :

$(\mathrm{d} c / \mathrm{d} N)_{w}=C_{\mathrm{p}}(\mathrm{d} c / \mathrm{d} N)_{\text {ref }}$ avec :

$(\mathrm{d} c / \mathrm{d} N)_{w}$ : vitesse de fissuration avec expansion

$(\mathrm{d} c / \mathrm{d} N)_{\text {ref }}$ : vitesse de fissuration sans expansion d'où :

avec :

$$
\begin{aligned}
C_{\mathrm{p}} & =\left(\Delta K_{\mathrm{eff}, w, 2 \mathrm{D}} / \Delta K_{\mathrm{eff}, \mathrm{ref}, 2 \mathrm{D}}\right)^{m} \\
& =\left[\left(1-\alpha_{R_{\mathrm{s}}}\right) w /\left(1-\alpha_{R}\right)\right]^{m}
\end{aligned}
$$

$\alpha_{R}=K_{\mathrm{op}} / K_{w, 2 \mathrm{D}}=1-U(R)(1-R)$

$U(R)=A+B R$, relation d'Elber, $A$ et $B$ étant des constantes

$m$, exposant de la loi de Paris

$\rightarrow$ Données d'entrée : loi de comportement en fissuration et en fermeture du matériau.

\section{Étape $3 b$}

Prévision de la fissuration, fissure de coin, soumise à la fois aux contraintes résiduelles et aux contraintes mécaniques de vol.

- Calcul de facteur d'intensité de contrainte $K_{w, 3 \mathrm{D}}$ pour un défaut coin quart d'ellipse : $K_{w, 3 \mathrm{D}}=w K_{\text {ref,3D }}$, où $K_{\text {ref,3D }}$ est le facteur $K$ de la fissure quart d'ellipse sans la contribution des contraintes résiduelles, qui peut être calculé de manière classique.

- Calcul de $\Delta K_{\text {eff }, w, 3 \mathrm{D}}=K_{w, 3 \mathrm{D}}-K_{\text {op }}$, en utilisant la relation d'Elber, adaptée éventuellement aux petites fissures, et le rapport $R_{\mathrm{s}}$.

- Application de la loi de fissuration du matériau de base (coefficients $C_{\text {eff }}$ et exposant $m$ ) pour prédire la vitesse avec l'effet de retard dû au procédé : $(\mathrm{d} c / \mathrm{d} N)_{w}=C_{\text {eff }}\left(\Delta K_{\text {eff }, w, 3 \mathrm{D}}\right)^{m}, c$ étant la longueur de la fissure en surface.

Remarque : la vitesse de fissuration peut aussi être calculée à partir du coefficient de retard calculé en étape 3a et de $K_{\text {ref,3D. }}$.

$\rightarrow$ Données d'entrée : loi de comportement en fissuration et en fermeture du matériau, longueur de fissure, rapport de forme $a / c$ de la fissure de coin.

\section{Remarque plus générale}

L'expression de $\alpha_{R}$ peut être enrichie en y intégrant l'effet petite fissure, à savoir que le niveau d'ouverture de la fissure dépend de la longueur de celle-ci aux faibles valeurs [7] :

$$
\alpha_{R}=[1-U(R)(1-R)]\left(1-e^{-k a}\right) .
$$

\section{Matériaux étudiés et matrice de travaux}

Les alliages étudiés sont les alliages d'aluminium 2024T351 et 7075T651, sens L-T, en épaisseur 2 et $5 \mathrm{~mm}$. Leurs principales caractéristiques mécaniques sont données en tableau 1 [3]. Les paramètres d'ouverture pour 
Tableau 1. Caractéristiques mécaniques des alliages 2024T351 et 7075T651 [3].

\begin{tabular}{cccccccc}
\hline & \multicolumn{2}{c}{ Caractéristiques statiques (sens L) } & \multicolumn{2}{c}{$\mathrm{d} a / \mathrm{d} N=C_{\text {eff }}\left(\Delta K_{\text {eff }}\right)^{m}, \mathrm{~L}-\mathrm{T}$} & \multicolumn{2}{c}{ Paramètres de fermeture } \\
\cline { 2 - 7 } & $E(\mathrm{MPa})$ & $R_{\mathrm{e}}(\mathrm{MPa})$ & $R_{\mathrm{m}}(\mathrm{MPa})$ & $C_{\text {eff }}$ & $m$ & $A$ & $B$ \\
\hline 2024T351 & 68421 & 368 & 580 & $4,16 \times 10^{-7}$ & 3,06 & 0,55 & 0,45 \\
7075T651 & 66027 & 550 & 675 & $8,32 \times 10^{-7}$ & 2,58 & 0,78 & 0,22 \\
\hline
\end{tabular}

Tableau 2. Matrice des travaux.

\begin{tabular}{|c|c|c|c|c|}
\hline \multirow[t]{2}{*}{ cas } & \multicolumn{3}{|c|}{ essai } & \multirow[t]{2}{*}{ calculs } \\
\hline & $\begin{array}{l}\text { matériau } \\
\text { épaisseur }\end{array}$ & $\begin{array}{c}\text { Conditions } \\
R \text { et } S_{\max } \\
\text { (pleine section) }\end{array}$ & Éprouvette & \\
\hline référence & $\begin{array}{c}2024 \\
2 \mathrm{~mm} \\
7075 \\
2 \mathrm{~mm}\end{array}$ & 0,1 et $150 \mathrm{MPa}$ & $\begin{array}{c}\text { Type } \mathrm{T} \\
\text { (Trou libre) }\end{array}$ & $\begin{array}{l}\text { Vérification de la méthode sur } \\
\text { éprouvette de référence sans effet } \\
\text { technologique }\end{array}$ \\
\hline interférence & $\begin{array}{c}2024 \\
2 \mathrm{~mm} \\
7075 \\
2 \mathrm{~mm} \\
\end{array}$ & 0,1 et $150 \mathrm{MPa}$ & $\begin{array}{c}\text { Type } \mathrm{T} \text { avec pion } \\
\text { monté en } \\
\text { interférence }\end{array}$ & Validation de la méthode \\
\hline $\begin{array}{c}\text { expansion non } \\
\text { habitée }\end{array}$ & $\begin{array}{c}2024 \\
5 \mathrm{~mm} \\
7075 \\
5 \mathrm{~mm}\end{array}$ & 0,1 et $170 \mathrm{MPa}$ & Type T & Validation de la méthode \\
\hline $\begin{array}{l}\text { expansion } \\
\text { habitée }\end{array}$ & $\begin{array}{c}2024 \\
5 \mathrm{~mm}\end{array}$ & 0,1 et $75 \mathrm{MPa}$ & Type Trou Habité & Validation de la méthode \\
\hline $\begin{array}{c}\text { expansion } \\
+ \\
\text { interférence }\end{array}$ & 7075 & Pas & d'essai & $\begin{array}{l}\text { Application de la méthode pour } \\
\text { des calculs prospectifs d'aide à la } \\
\text { justification en tolérance aux } \\
\text { dommages de jonctions rivetées }\end{array}$ \\
\hline
\end{tabular}

les petites fissures sont $k=10 \mathrm{~mm}^{-1}$ pour le 2024 et $k=1,59 \mathrm{~mm}^{-1}$ pour le $7075, a$ étant la longueur de la fissure en $\mathrm{mm}$ [7].

Le tableau 2 présente la matrice des cas abordés en calculs et essais. Plus de détails sont données dans la suite du texte.

\section{Cas considéré comme référence $[4,5]$}

\subsection{Cas étudiés (Tab. 2)}

L'éprouvette $\mathrm{T}$ est une plaque rectangulaire de hauteur $H=250 \mathrm{~mm}$, de largeur $W=50 \mathrm{~mm}$, d'épaisseur $B=2 \mathrm{~mm}$, avec un trou central de diamètre $D=$ 6,22 mm (Fig. 3). Un défaut initial traversant de longueur $c_{0}=0,2 \mathrm{~mm}$ est usiné à la scie d'horloger, à mi-hauteur, sur un côté de l'alésage. L'orientation de l'éprouvette est L-T. La contrainte maximale, du cycle de fatigue appliqué, a pour valeur $S_{\max }=150 \mathrm{MPa}$, relativement à la section pleine de l'éprouvette, pour les deux alliages. La longueur de fissure est suivie en surface à l'aide d'un microscope optique, de résolution $0,01 \mathrm{~mm}$, installé sur un vernier.

\subsection{Résultats de la méthode prévisionnelle (étape 2)}

Les calculs issus de l'étape 2 sont présentés ici pour les deux alliages. Le calcul du facteur $K$ par la méthode montre une concordance satisfaisante avec la formule analytique classique (code ESACRAK [8]) puisque l'on constate moins de $6 \%$ de différence (Fig. 4 et Tab. 3), sauf pour la plus faible longueur de fissure de $0,1 \mathrm{~mm}$ pour laquelle l'écart vaut $40 \%$ pour le 2024 et $25 \%$ pour le 7075. À cela, deux explications peuvent être avancées (Tab. 4) : une taille de zone plastique non négligeable devant la longueur de la fissure et une singularité différente de $-1 / 2$ est constatée sur les résultats des calculs, ce qui remettrait en cause l'application de la formulation analytique qui est purement élastique. Noter que l'on retrouverait le rayon des zones plastiques en appliquant la formule d'Irwin, pour une longueur de fissure supérieure à $0,5 \mathrm{~mm}$. La figure 5 présente la distribution du champ des contraintes $S_{22}$ (perpendiculaires à la fissure) en avant de l'alésage sous chargement maxi et mini et le rapport $R_{\mathrm{S}}$ «stabilisé » au loin de l'alésage dans le cas du 7075. On retrouve bien le rapport $R$ appliqué de 0,1 . La même valeur du rapport $R_{\mathrm{S}}$ est obtenue pour le 2024 . 


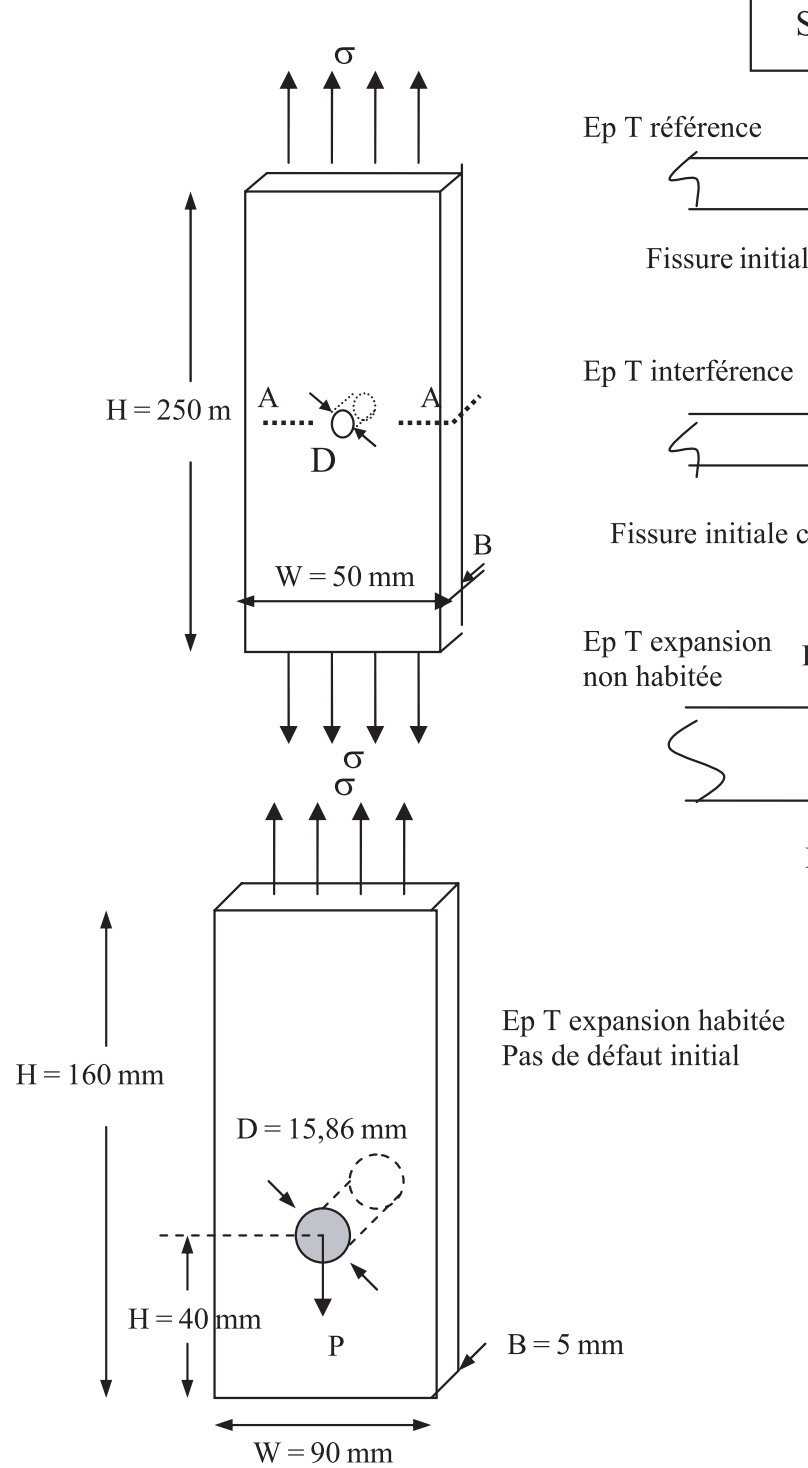

Section A-A

$\mathrm{D} \stackrel{6,22 \mathrm{~mm}}{\longrightarrow}$

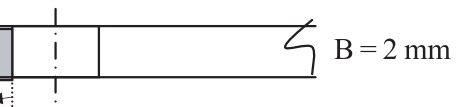

$\stackrel{\circ}{=} 0,2 \mathrm{~mm}$

$\mathrm{D} \underset{\longleftarrow}{\stackrel{6}{\stackrel{6}{\longrightarrow}} \rightarrow 32} \mathrm{~m}$

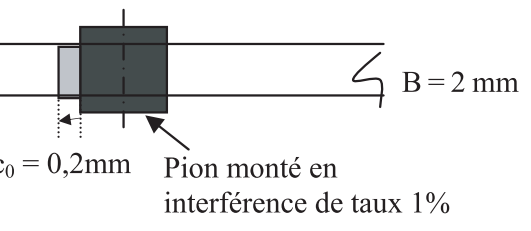

Ep T expansion $\quad \mathrm{D}=7,37 \mathrm{~mm}$ et $7,42 \mathrm{~mm}$

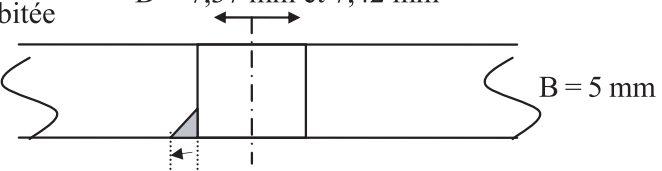

Fissure initiale $\mathrm{c}_{0}$

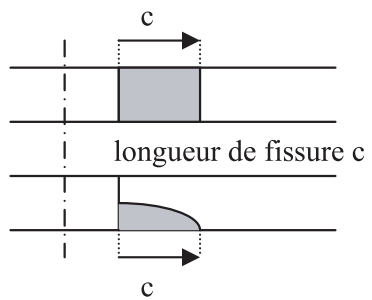

Fig. 3. Géométrie des éprouvettes d'essai : cas de référence, cas d'interférence, cas d'expansion, cas d'expansion trou habité.

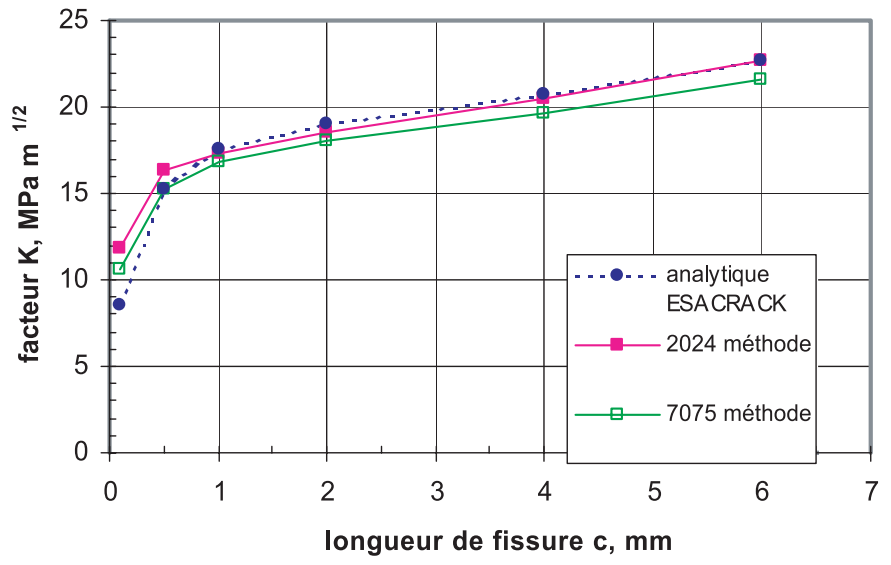

Fig. 4. Cas de référence pour les alliages 2024 et 7075 . Application de la méthode (étape 2) pour le calcul du facteur $K$ en fonction de la longueur de fissure et comparaison avec des résultats de calculs analytiques.
Tableau 3. Cas de référence. Valeurs de facteurs $K_{\text {ref,2D }}$, étape 2 de la méthode $\left(S_{\max }=150 \mathrm{MPa}\right)$.

\begin{tabular}{cccc}
\hline \multirow{2}{*}{$\begin{array}{c}\text { Longueur } \\
\text { de fissure }\end{array}$} & Calcul analytique & \multicolumn{2}{c}{ Méthode prévisionnelle } \\
\cline { 3 - 4 } \multicolumn{1}{c}{ code ESACRACK $[8])$} & 2024 & 7075 \\
\hline$(\mathrm{mm})$ & $K_{\mathrm{ref}, 2 \mathrm{D}}$ & $K_{\mathrm{ref}, 2 \mathrm{D}}$ & $K_{\mathrm{ref}, 2 \mathrm{D}}$ \\
\hline 0,1 & $(\mathrm{MPa} \sqrt{m})$ & $(\mathrm{MPa} \sqrt{m})$ & $(\mathrm{MPa} \sqrt{m})$ \\
0,5 & 8,48 & 11,88 & 10,62 \\
1 & 15,26 & 16,30 & 15,23 \\
2 & 17,61 & 17,37 & 16,80 \\
4 & 19,07 & 18,48 & 17,99 \\
6 & 20,77 & 20,48 & 19,66 \\
\hline
\end{tabular}



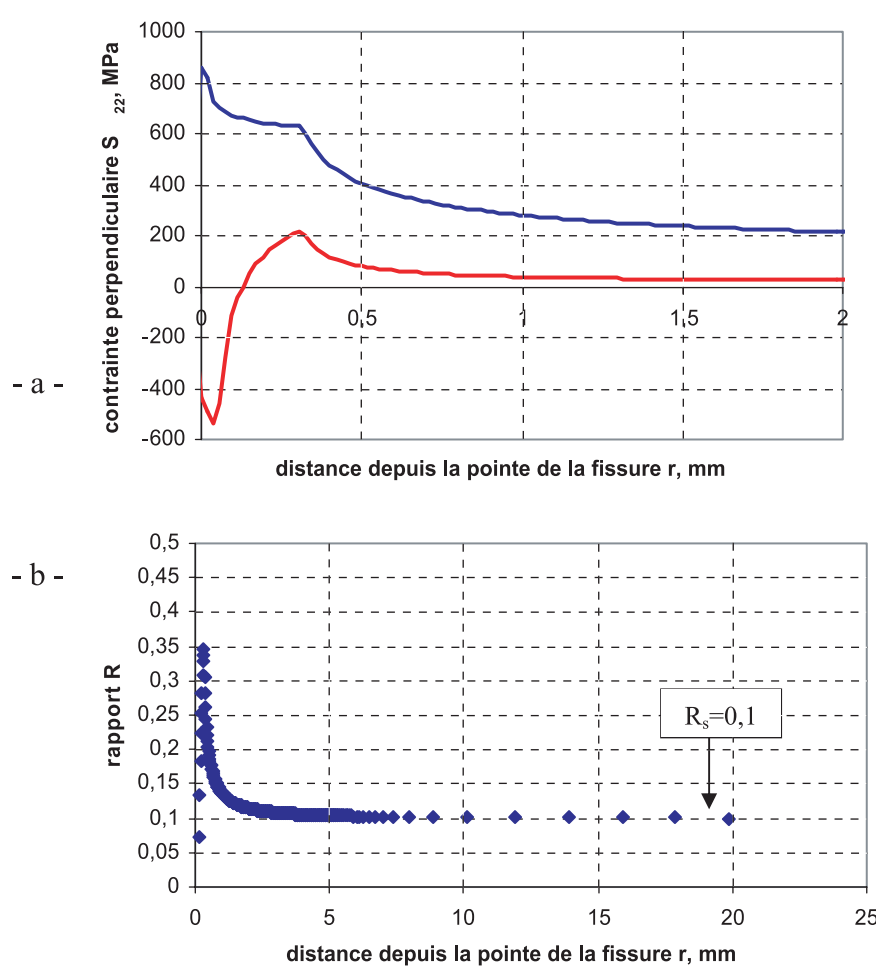

Fig. 5. Cas de référence pour l'alliage 7075 , étape 2 de la méthode. (a) Champ des contraintes $S_{22}$, perpendiculaires au plan de fissuration et selon le plan médian, valeurs maximales et minimales en avant de la pointe d'une fissure de longueur $2 \mathrm{~mm}$. (b) Détermination du rapport $R$ vu par la fissure. Ce dernier est constant au loin de l'alésage, et vaut $R_{\mathrm{s}}=0,1$ ce qui est le rapport $R$ appliqué.

Tableau 4. Cas de référence. Rayon $r_{\mathrm{p}}$ de la zone plastique.

\begin{tabular}{lllllll}
\hline \multirow{2}{*}{$\begin{array}{l}\text { Exploitation } \\
\text { calculs EF }\end{array}$} & 0,10 & 0,50 & 1,00 & 2,00 & 4,00 & 6,00 \\
\hline \cline { 2 - 7 } 2024, $r_{\mathrm{p}}(\mathrm{mm})$ & 0,15 & 0,31 & 0,35 & 0,40 & 0,49 & 0,61 \\
$7075, r_{\mathrm{p}}(\mathrm{mm})$ & 0,05 & 0,12 & 0,15 & 0,18 & 0,21 & 0,25 \\
singularité & $-0,2$ & $-0,5$ & $-0,5$ & $-0,5$ & $-0,5$ & $-0,5$ \\
\hline
\end{tabular}

\subsection{Résultats d'essais et validation de la méthode (étape 3a)}

Un essai sur le 2024 et deux essais sur le 7075 ont été réalisés. Les vitesses de fissuration sont les vitesses sécantes calculées sur deux points de mesure successifs. La fissure s'est amorcée d'un côté de l'alésage sur le défaut initial, comme prévu, et a tout de suite pris l'allure d'une fissure traversante. Les calculs de facteur $K$, obtenus par la méthode décrite dans la section 3.2 , ont permis de faire des prévisions de la vitesse de fissuration (étape 3a). Les figures 6 et 7 montrent un accord satisfaisant entre essais et calculs. Toutefois le point correspondant à $0,1 \mathrm{~mm}$ reste à confirmer puisque les essais commençaient avec une fissure de longueur supérieure à $0,2 \mathrm{~mm}$.

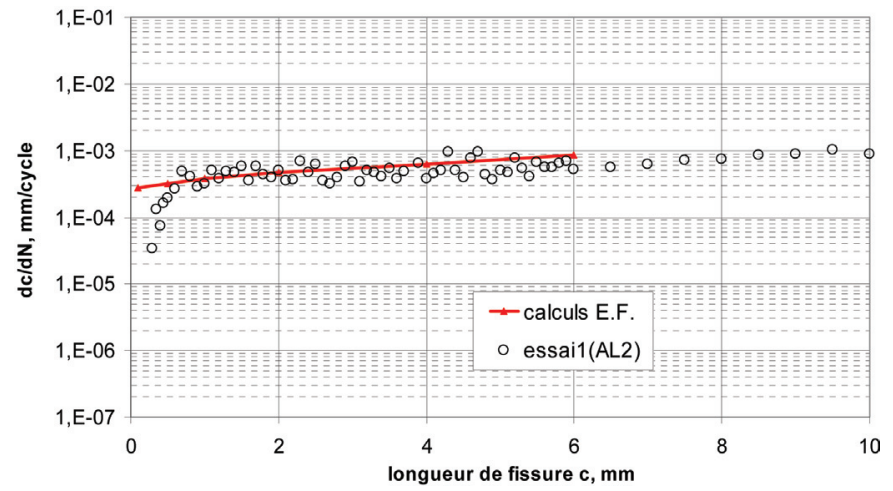

Fig. 6. Cas de référence pour l'alliage 2024. Vitesse de fissuration en fonction de la longueur de fissure : comparaison des prévisions par calculs (étape 3a) avec les résultats d'essai.

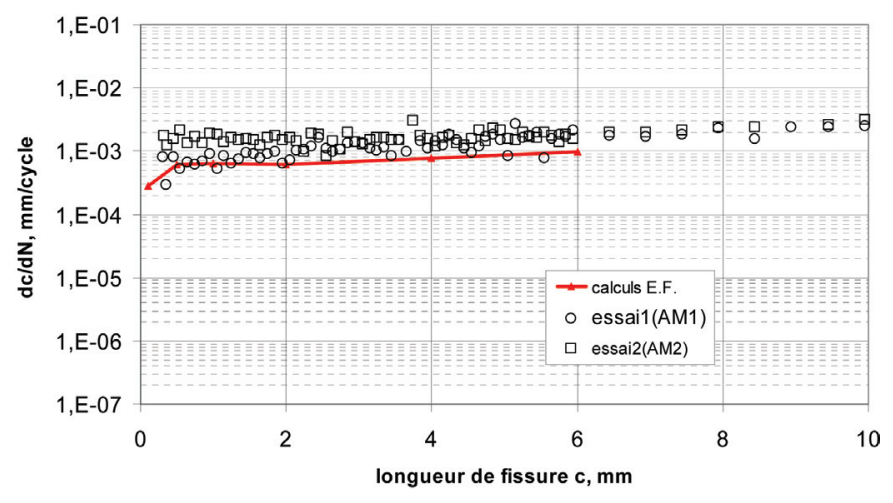

Fig. 7. Cas de référence pour l'alliage 7075. Vitesse de fissuration en fonction de la longueur de fissure : comparaison des prévisions par calculs (étape 3a) avec les résultats d'essais.

\subsection{Conclusion}

La méthode est vérifiée pour le calcul des facteurs $K$ de référence.

\section{Cas d'une interférence [5]}

\subsection{Cas examinés (Tab. 2)}

L'éprouvette $\mathrm{T}$ est une plaque rectangulaire de hauteur $H=250 \mathrm{~mm}$, de largeur $W=50 \mathrm{~mm}$, d'épaisseur $B=2 \mathrm{~mm}$, avec un trou central habité par un fût de rivet en alliage de titane monté en interférence (taux de $1 \%$, cas d'une forte interférence), de diamètre $D=6,32 \mathrm{~mm}$ en présence du pion (Fig. 3). Un défaut initial traversant de longueur $c_{0}=0,2 \mathrm{~mm}$ est usiné à la scie d'horloger, à mi-hauteur, sur un côté de l'alésage, avant la pose du pion. L'orientation de l'éprouvette est L-T, la contrainte maximale, brute de section, de fatigue $S_{\max }$ appliquée a pour valeur $150 \mathrm{MPa}$ sur les deux alliages. La longueur de fissure est suivie en surface à l'aide d'un microscope optique, de résolution $0,01 \mathrm{~mm}$, installé sur un vernier. 


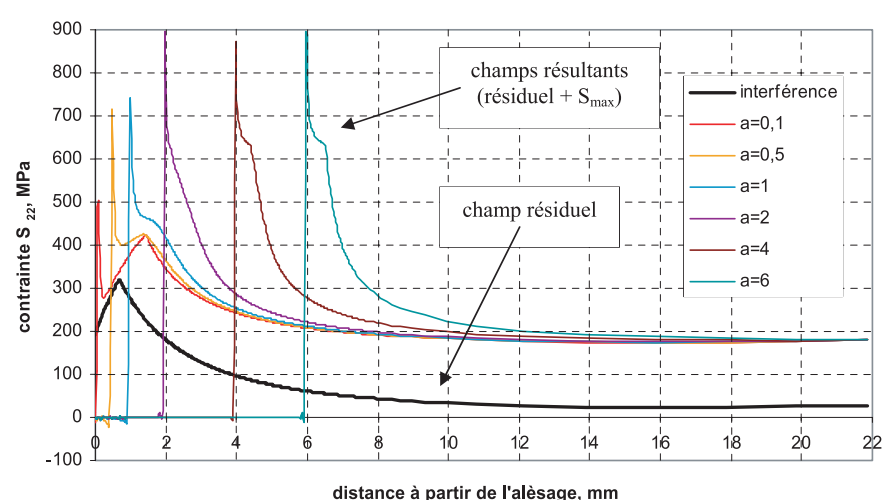

Fig. 8. Cas d'interférence pour l'alliage 7075. Évolution de la contrainte $S_{22}$, selon le plan médian, en fonction de la distance à partir du bord de l'alésage : champ de contraintes résiduelles dues à l'interférence, champ de contraintes résultant de la combinaison des contraintes résiduelles et de la sollicitation $S_{\max }$ de fatigue de $150 \mathrm{MPa}$ pour différentes longueurs de fissures traversantes $(0,1 ; 0,5 ; 1 ; 2 ; 4$ et $6 \mathrm{~mm})$.

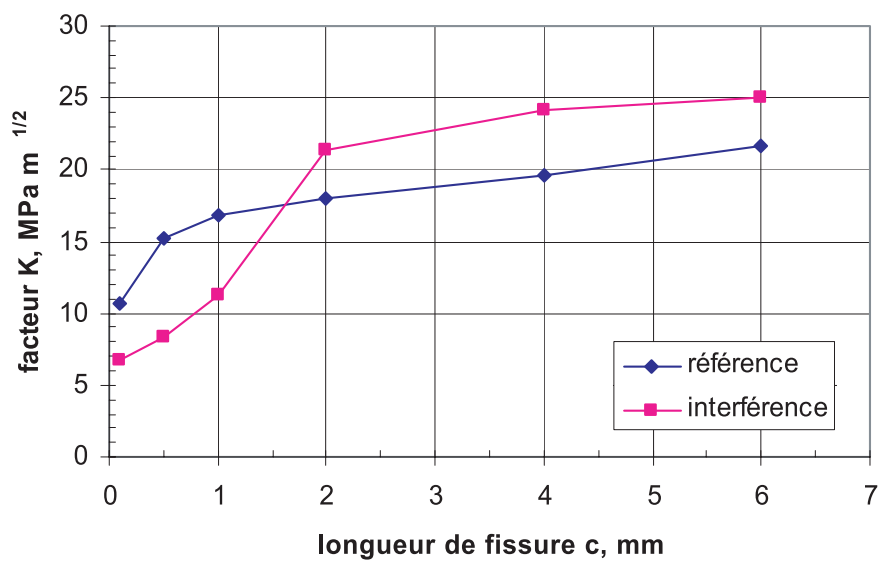

Fig. 9. Cas d'interférence, alliage 7075. Calcul du facteur $K$ en fonction de la longueur de fissure et comparaison avec le cas de référence (étape 2 de la méthode).

\subsection{Résultats de la méthode prévisionnelle (étape 2)}

Les calculs concernent une fissure traversante située d'un côté de l'alésage. La figure 8 présente les champs de contraintes $S_{22}$, selon le plan médian de l'éprouvette, en avant de l'alésage, pour le 7075 : d'une part le champ résiduel dû à l'interférence (sans fissure), d'autre part celui résultant de ce dernier et de l'application de la contrainte maximale de fatigue, pour plusieurs longueurs de fissure jusqu'à $6 \mathrm{~mm}$. Noter que les contraintes résiduelles sont de traction.

L'analyse des champs de contrainte a permis de déterminer la singularité élastique et les résultats sont consignés dans le tableau 5 pour les deux alliages.

La figure 9 montre une comparaison avec le cas de référence, pour le 7075. L'interférence a pour effet de diminuer le facteur $K$ aux faibles longueurs de fissures (jusqu'à $2 \mathrm{~mm}$ ), puis de l'augmenter au-delà. Le rapport $R_{\mathrm{S}}$ stabilisé vu au loin par la fissure est de 0,22 pour toutes les longueurs de fissure. Il est différent du rapport appliqué

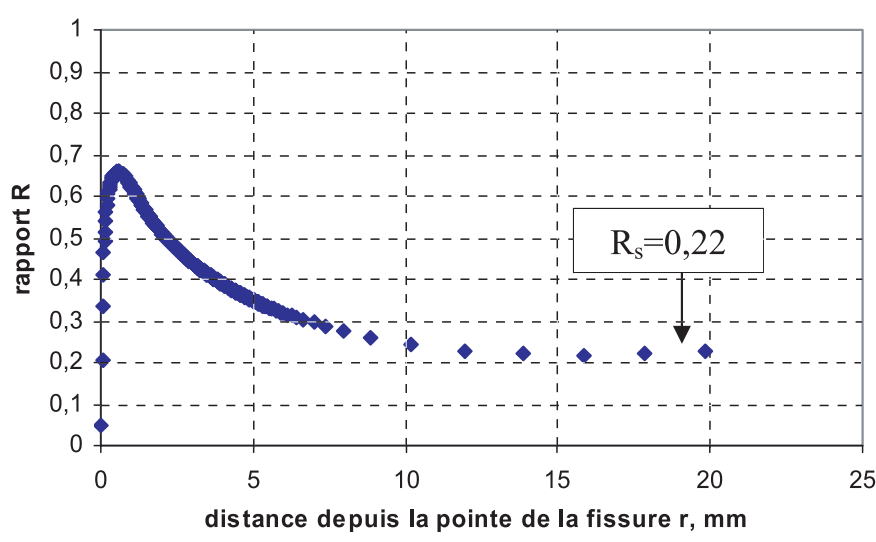

Fig. 10. Cas d'interférence, alliage 7075. Détermination du rapport $R$ vu par une fissure de longueur de $2 \mathrm{~mm}$ (étape 2 de la méthode). Ce rapport se stabilise à une valeur $R_{\mathrm{s}}$ de 0,22 , il est différent de celui appliqué de 0,1 .

Tableau 5. Cas d'une interférence. Valeurs de facteurs $K_{w, 2 \mathrm{D}}$, étape 2 , et coefficient de retard $C_{\mathrm{p}}$, étape 3 a de la méthode $\left(S_{\max }=150 \mathrm{MPa}\right)$. Les calculs concernent une fissure traversante, située d'un côté de l'alésage et de longueur en surface $c$.

\begin{tabular}{ccccc}
\hline \multirow{2}{*}{$\begin{array}{c}\text { Longueur } \\
\text { de fissure }\end{array}$} & \multicolumn{4}{c}{ Méthode prévisionnelle } \\
\cline { 2 - 5 }$c$ & \multicolumn{2}{c}{2024} & \multicolumn{3}{c}{7075} \\
$(\mathrm{~mm})$ & $\begin{array}{c}K_{w, 2 \mathrm{D}} \\
(\mathrm{MPa} \sqrt{m})\end{array}$ & $C_{\mathrm{p}}$ & $\begin{array}{c}K_{w, 2 \mathrm{D}} \\
(\mathrm{MPa} \sqrt{m})\end{array}$ & $C_{\mathrm{p}}$ \\
\hline 0,1 & 4,47 & 0,047 & 6,69 & 0,21 \\
0,5 & 5,55 & 0,031 & 8,37 & 0,17 \\
1 & 7,40 & 0,063 & 11,23 & 0,29 \\
2 & 11,81 & 0,22 & 21,35 & 1,18 \\
4 & 22,53 & 1,13 & 24,17 & 1,26 \\
6 & 25,16 & 1,16 & 25,05 & 1,08 \\
\hline
\end{tabular}

de 0,1 , ce qui traduit un autre effet de l'interférence. La figure 10 montre ce rapport pour une fissure de longueur $2 \mathrm{~mm}$ sur le 7075 .

Pour le 2024, on trouve le même rapport $R$ stabilisé de 0,22 mais des valeurs de facteur $K$ plus faibles (Tab. 5). À noter toutefois qu'aux faibles longueurs de fissure, le facteur $K$ semble rester constant dans le cas du 2024 mais pas du 7075 .

\subsection{Résultats d'essais et validation de la méthode (étape 3b)}

Un essai sur le 2024 et deux essais sur le 7075 ont été réalisés. Dans chaque cas, la fissure s'est amorcée sur un des deux coins du défaut initial, comme prévu. La fissure s'est propagée d'abord en défaut coin de type quart d'ellipse $(a, c)$ puis est devenue une fissure traversante lorsque la longueur en surface $c$ a atteint $6 \mathrm{~mm}$ (Fig. 11, [3]).

Les figures 12 et 13 montrent l'évolution de la vitesse de fissuration avec la longueur de fissure en surface $c$. Les vitesses sont les vitesses sécantes calculées sur deux points de mesure successifs. La vitesse de fissuration ne cesse 


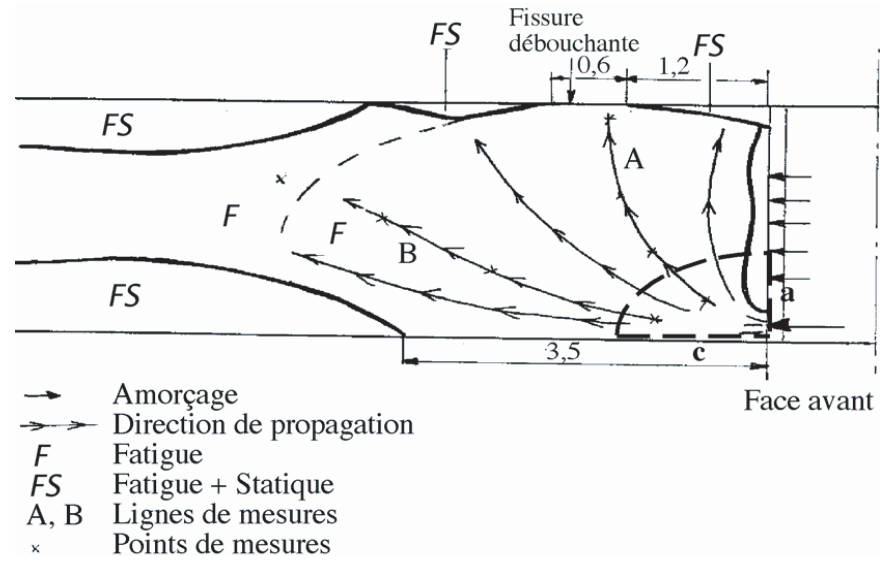

Fig. 11. Cas d'interférence, alliage 7075. Fractographie : fissure en défaut coin quart d'ellipse $(a, c)$ au départ avant traversée de l'épaisseur pour devenir une fissure traversante.

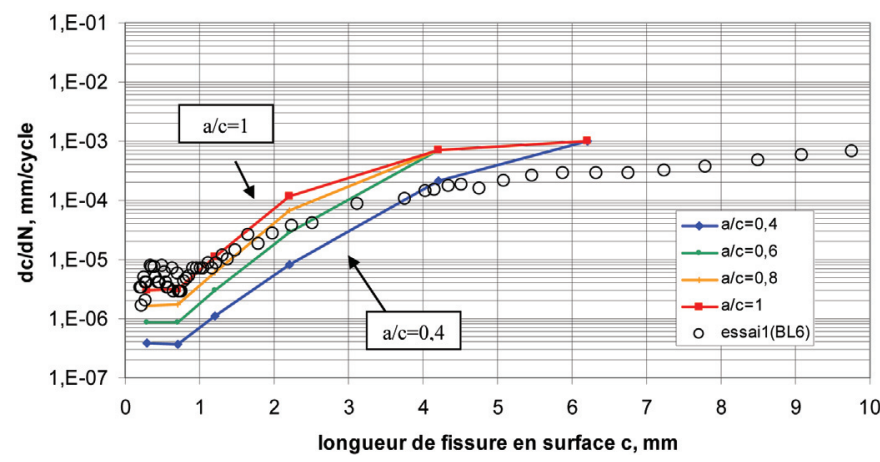

Fig. 12. Cas d'interférence, alliage 2024. Vitesse de fissuration en fonction de la longueur de fissure en surface : résultats d'essais et calculs prévisionnels pour différents rapports de forme $a / c$ (étape $3 \mathrm{~b}$ de la méthode).

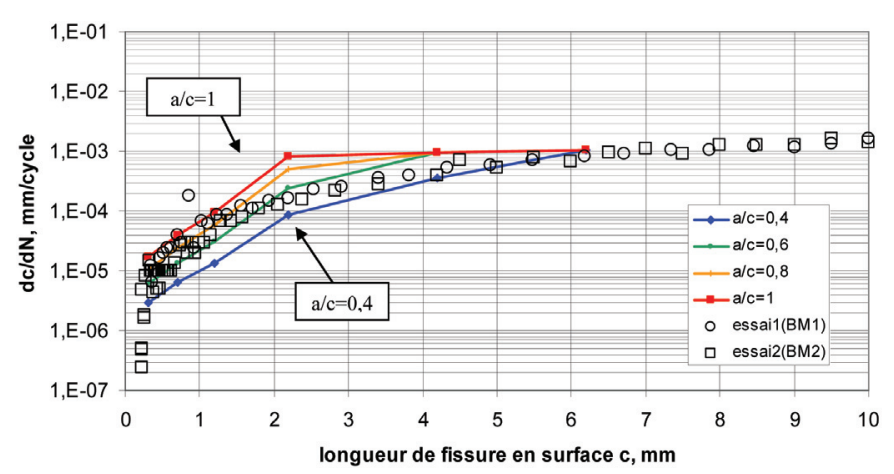

Fig. 13. Cas d'interférence, alliage 7075 . Vitesse de fissuration en fonction de la longueur de fissure en surface : résultats d'essais et calculs prévisionnels pour différents rapport de forme $a / c$ (étape $3 \mathrm{~b}$ de la méthode).

d'augmenter pour atteindre un niveau constant lorsque la fissure atteint une longueur en surface égale à une fois le diamètre de l'alésage, qui est la distance à partir de laquelle les contraintes résiduelles se stabilisent à un niveau bas.

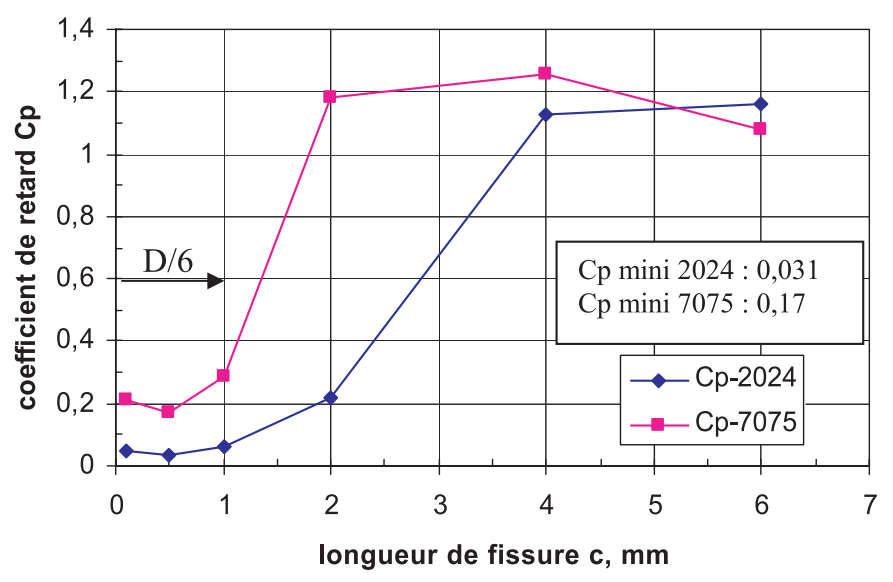

Fig. 14. Cas d'interférence, alliages 2024 et 7075 . Abaques de retard : coefficient de retard $C_{\mathrm{p}}$ en fonction de la longueur de fissure en surface (étape $3 a$ de la méthode). $D$ est le diamètre de l'alésage.

L'examen du faciès de fatigue a permis d'estimer le rapport de forme $a / c$, où $a$ est la longueur dans l'alésage et $c$ la longueur en surface, qui est variable : $a / c$ est proche de 1 au départ et chute à 0,4 lorsque la fissure atteint une longueur en surface $c$ de 4 à $5 \mathrm{~mm}$, pour les deux alliages. Les calculs de l'étape $3 \mathrm{~b}$ ont donc été réalisés avec différentes valeurs de $a / c$ (de $0,4,0,6,0,8$ et 1 ), en fonction de la longueur de fissure en surface $c$. Pour les calculs de facteur $K$ pour le défaut coin, la présence du défaut initial a été négligée du fait de sa faible profondeur en regard du diamètre de l'alésage.

Les figures 12 et 13 présentent les résultats expérimentaux et leur comparaison avec les calculs effectués pour des fissures en forme de quart d'ellipse qui approchent le défaut réel. En se déplaçant du réseau $a / c=1$ à $a / c=0,4$, de $c=0$ à $c=4-5 \mathrm{~mm}$, les calculs prévisionnels reproduisent de manière satisfaisante le comportement de la fissure. De plus les résultats d'essais confirment la constance du facteur $K$ pour le 2024 aux faibles longueurs de fissure.

Cet effet de constance, constaté pour le 2024 mais pas pour le 7075, n'aurait pas pu être prévu par des approches purement élastiques cités en référence car les profils de contraintes résiduelles sont similaires pour les deux matériaux.

Des abaques de retard ont été construits à partir du calcul 2D, en divisant la vitesse prédite avec interférence par celle prédite pour le cas de référence (Fig. 14). Les abaques montrent un effet retard important (facteur 6 pour le 7075 et 30 pour le 2024). Le retard maximal est atteint aux environs d'une distance $D / 6$ à partir du bord de l'alésage. Cet abaque est aussi valable aussi pour une fissure de coin puisque le terme $w$ se conserve.

\subsection{Conclusion}

La méthode est vérifiée pour le calcul des facteurs $K$ avec la prise en compte de la présence de contraintes résiduelles d'interférence. 


\section{Cas d'une expansion [4]}

\subsection{Cas d'étude}

Les cas examinés sont les suivants :

1. Alésage expansé, non habité, pour le 2024 et le 7075 :

$\rightarrow$ L'éprouvette $\mathrm{T}$ est une plaque rectangulaire, de largeur $W=50 \mathrm{~mm}$, de hauteur $H=250 \mathrm{~mm}$, d'épaisseur $B=5 \mathrm{~mm}$, avec un trou central de diamètre après expansion $D=7,42 \mathrm{~mm}$ pour le 2024 et $D=7,37 \mathrm{~mm}$ pour le 7075 (taux d'expansion de $4 \%$ sur un diamètre initial de $7,24 \mathrm{~mm}$ ). Un défaut initial est réalisé en coin d'alésage, avec une longueur de 0,26 mm, sur la face de l'éprouvette correspondant à la face d'entrée du brunissoir (Fig. 3). L'effort de fatigue est appliqué aux extrémités de l'éprouvette, la contrainte maximale, brute de section, est $170 \mathrm{MPa}$, et le rapport de chargement vaut $R=0,1$.

2. Alésage expansé habité, cas représentatif d'une jonction avec un coefficient de transfert d'effort de $100 \%$, pour le 2024 seulement :

$\rightarrow$ L'éprouvette est une plaque rectangulaire, de largeur $W=90 \mathrm{~mm}$, de hauteur $H=160 \mathrm{~mm}$, d'épaisseur $B=5 \mathrm{~mm}$, avec un trou de diamètre après réalésage $D=15,86 \mathrm{~mm}$ (taux d'expansion de $4 \%$ sur un diamètre initial de $15,20 \mathrm{~mm}$, donnant un diamètre après expansion de 15,60 mm qui est réalésé), sans défaut initial en bord d'alésage. Le centre du trou est situé à mi-largeur et à $h=40 \mathrm{~mm}$ d'un bord de l'éprouvette. L'effort de fatigue est appliqué sur le trou au moyen d'une goupille et à l'extrémité de l'éprouvette la plus éloignée, la contrainte maximale de fatigue vaut $75 \mathrm{MPa}$, relativement à la section pleine de l'éprouvette, et le rapport de chargement $R$ vaut 0,1 . La goupille est introduite sans interférence avec un léger jeu (Fig. 3).

\subsection{Cas de l'alésage expansé non habité : essais et calculs étapes 2 et $3 b$}

La figure 15 présente le champ de contrainte issu de l'étape 2, sur l'axe de fissuration et perpendiculairement à ce dernier, pour une fissure de longueur $1 \mathrm{~mm}$, pour le 7075 : contraintes résiduelles après expansion et création de la fissure dans le maillage (la création de la fissure ne perturbe pas le champ résiduel), champ résultant de l'application de la contrainte de fatigue $S_{\max }=170 \mathrm{MPa}$ sur le champ résiduel, champ résultant de l'application de la contrainte de fatigue $S_{\min }=17 \mathrm{MPa}$ sur le champ résiduel. Des conditions aux limites ont été introduites pour éviter aux lèvres de la fissure de s'interpénétrer. La valeur minimale de la contrainte résiduelle est située à une distance $D / 6$ du bord de l'alésage. Noter que la contrainte résiduelle ne chute pas vers zéro lorsque l'on s'éloigne de l'alésage, ce qui conduit à un rapport $R$ stabilisé $R_{\mathrm{s}}$ de 0,2 pour toutes les longueurs de fissures. Le tableau 6 consigne les valeurs de facteur $K$ obtenus par la méthode. La comparaison avec le cas sans expansion est illustrée par la fissure 16 pour le 7075 . L'approche citée en [1] a

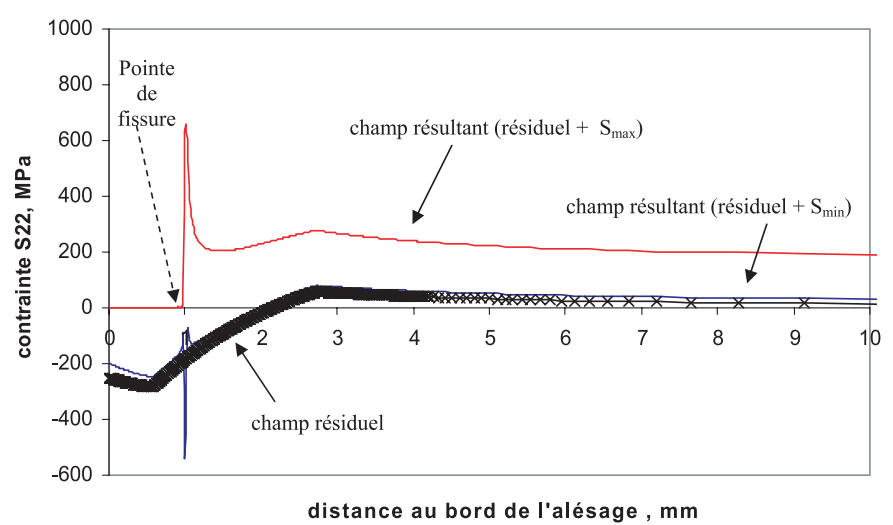

Fig. 15. Cas d'expansion non habitée, alliage 7075. Distribution des contraintes en pointe d'une fissure traversante de longueur $1 \mathrm{~mm}$ (étape 2 de la méthode) selon le plan médian : champ résiduel, champ résultant du résiduel et de l'application de la contrainte $S_{\max }$ de $170 \mathrm{MPa}$, champ résultant du résiduel et de l'application de la contrainte $S_{\min }$ de $17 \mathrm{MPa}$. Ici, le rapport stabilisé $R_{\mathrm{s}}$ est égal à 0,2 .

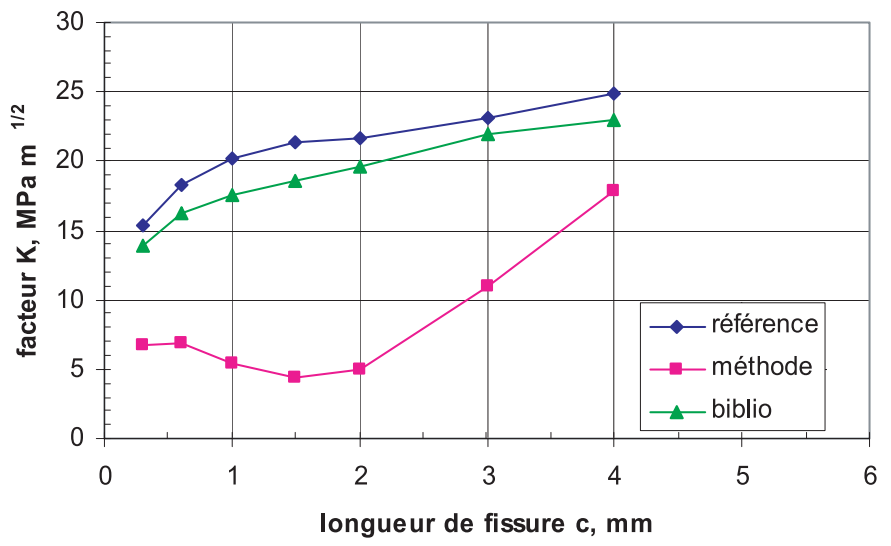

Fig. 16. Cas d'expansion non habitée, alliage 7075. Calcul du facteur $K$ en fonction de la longueur de fissure (étape 2 de la méthode) et comparaison avec le cas sans expansion et avec l'application de l'approche citée en bibliographie [1].

été mise en œuvre pour le cas du 7075 et les résultats sont consignés dans la figure 16. Cette approche prévoit peu d'effet retard car les valeurs de facteurs $K$ sont assez proches de celles du cas de référence.

Durant les essais, la fissure de fatigue s'est amorcée sur le défaut initial et s'est propagée en fissure coin. Le faciès de rupture a été examiné au microscope optique et au microscope électronique à balayage. Le facteur de forme n'était pas facile à déterminer, cependant l'examen a conclu aux identifications suivantes : pour le 2024, $a / c=$ 1 du début jusqu'à $c=4 \mathrm{~mm}$, pour le $7075, a / c=1$ au début et décroît jusqu'à 0,8 lorsque $c$ atteint 4 à $6 \mathrm{~mm}$. Les prévisions ont été faites pour les valeurs de $a / c$ suivantes : $0,4,0,5,0,6,0,7,0,8,0,9,1$. Les figures 17 et 18 montrent un bon accord entre les essais et les prévisions (étape $3 \mathrm{~b}$ ).

Il faut noter que la vitesse de fissuration est constante pour le 2024 durant le premier millimètre et demi de propagation, alors qu'elle diminue pour le 7075 pour ensuite croître après $1,5 \mathrm{~mm}$. La méthode suivie ici prévoit cet 


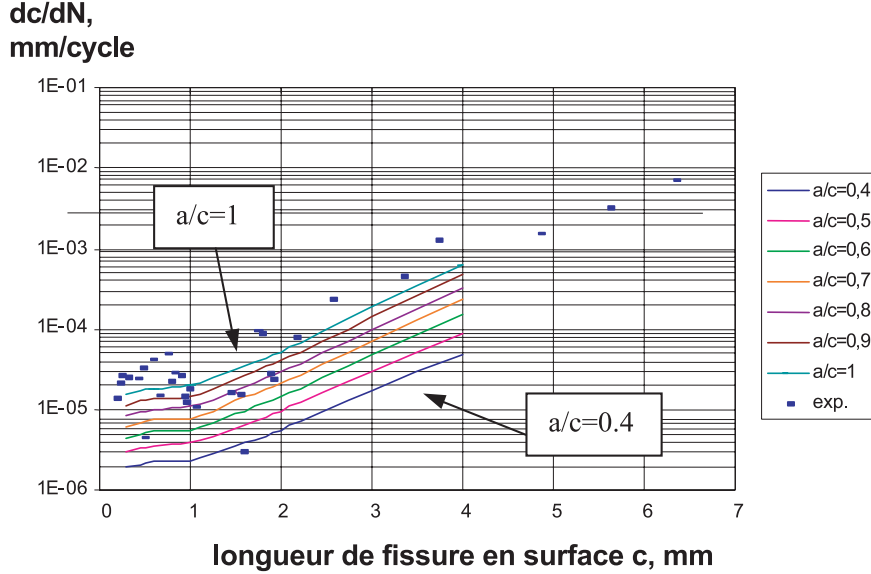

Fig. 17. Cas d'expansion non habitée, alliage 2024. Vitesse de fissuration en fonction de la longueur de fissure en surface : résultats d'essais et calculs prévisionnels pour différents rapport de forme $a / c$ (étape $3 \mathrm{~b}$ de la méthode).

$\mathrm{dc} / \mathrm{dN}$,

$\mathrm{mm} /$ cycle

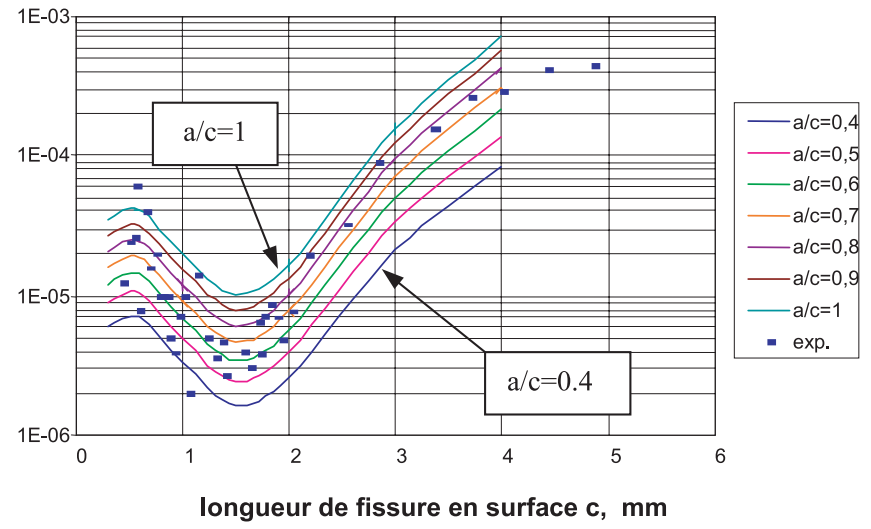

Fig. 18. Cas d'expansion non habitée, alliage 7075. Vitesse de fissuration en fonction de la longueur de fissure en surface : résultats d'essais et calculs prévisionnels pour différents rapport de forme $a / c$ (étape $3 \mathrm{~b}$ de la méthode).

effet alors que les approches purement élastiques cités en référence n'auraient pas pu le prédire puisque les profils des champs résiduels sont similaires pour les deux alliages.

Des jauges avaient été placées dans le voisinage de l'alésage, et leurs réponses suivies avant l'amorçage de la fissure : aucune relaxation des contraintes résiduelles, par fatigue, n'avait été détectée. Ce qui conforte l'approche qui supposait implicitement que les contraintes résiduelles ne se relâchaient pas.

Des abaques de retard ont été établis (Fig. 19) donnant le coefficient de retard $C_{\mathrm{p}}$ en fonction de la longueur de fissure en surface (Tab. 6, étape 3a). Les courbes ont une forme particulière, avec un minimum à une distance $D / 6$ du bord de l'alésage. Les abaques montrent un effet retard important (facteur 6 pour le 2024 et 70 pour le 7075$)$.

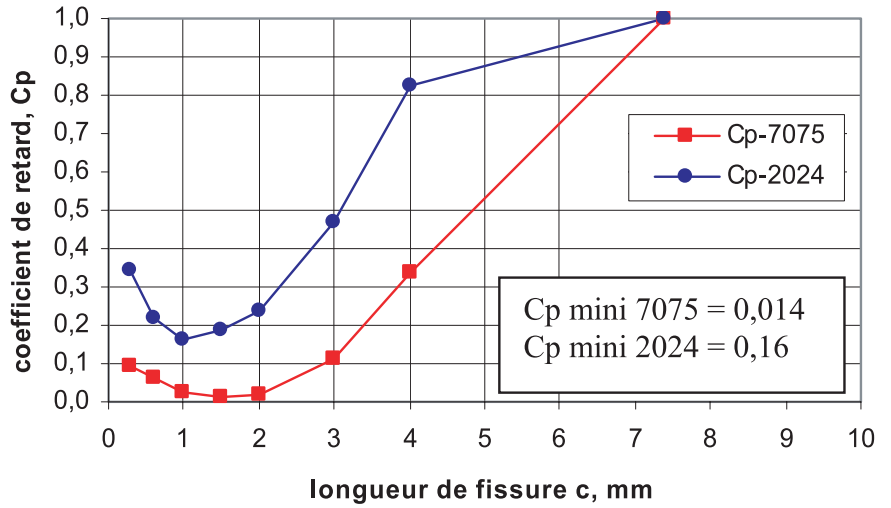

Fig. 19. Cas d'expansion non habitée, alliages 2024 et 7075 . Abaques de retard : coefficient de retard $C_{\mathrm{p}}$ en fonction de la longueur de fissure en surface $c$. $D$ est le diamètre de l'alésage.

Tableau 6. Cas d'une expansion non habitée. Valeurs de facteurs $K_{w, 2 \mathrm{D}}$, étape 2 , et coefficient de retard $C_{\mathrm{p}}$, étape 3a de la méthode $\left(S_{\max }=170 \mathrm{MPa}\right)$. Les calculs concernent une fissure traversante, située d'un côté de l'alésage et de longueur en surface $c$.

\begin{tabular}{ccccc}
\hline \multirow{2}{*}{$\begin{array}{c}\text { Longueur } \\
\text { de fissure }\end{array}$} & \multicolumn{4}{c}{ Méthode prévisionnelle } \\
\cline { 2 - 5 }$c$ & \multicolumn{2}{c}{2024} & \multicolumn{3}{c}{7075} \\
\hline$c$ & $K_{w, 2 \mathrm{D}}$ & $C_{\mathrm{p}}$ & $K_{w, 2 \mathrm{D}}$ & $C_{\mathrm{p}}$ \\
$(\mathrm{Mm})$ & $(\mathrm{MPa} \sqrt{m})$ & & 6,70 & 0,095 \\
\hline 0,3 & 10,59 & 0,34 & 6,85 & 0,061 \\
0,6 & 10,69 & 0,22 & 5,43 & 0,027 \\
1 & 10,56 & 0,16 & 4,42 & 0,014 \\
1,5 & 11,79 & 0,19 & 5,02 & 0,018 \\
2 & 13,02 & 0,24 & 11,00 & 0,11 \\
3 & 18,00 & 0,47 & 17,82 & 0,34 \\
4 & 24,17 & 0,82 & &
\end{tabular}

\subsection{Cas de l'alésage expansé habité : essais et calculs étapes 2 et 4}

Quatre fissures se sont amorcées à peu près en même temps, une en chaque coin de l'alésage. Après une avancée de 2 à $4 \mathrm{~mm}$, ces fissures coin ont coalescé pour donner naissance à deux fissures traversantes, une de chaque côté de l'alésage, quasi symétriques. L'analyse en mécanique de la rupture est restée $2 \mathrm{D}$ au-delà de $4 \mathrm{~mm}$. Les valeurs de $K$ obtenues (étape 2 ) sont consignées dans le tableau 7 . Le rapport $R_{\mathrm{S}}$ décroît de 0,39 à 0,23 lorsque $c$ croît de 0,3 à $16 \mathrm{~mm}$. Un calcul concernant le scénario de quatre fissures coin de forme quart d'ellipse et de rapport $a / c=$ 1 , symétriques de part et d'autre de l'alésage, mesurant 1,6 et $3 \mathrm{~mm}$ en surface, a été réalisé. Malgré la dispersion des résultats, toutes les prévisions sont en bon accord avec les essais (Fig. 20).

Le réalésage avait été simulé par éléments finis par enlèvement de matière. Il s'est avéré que cela ne changeait pas de manière significative le champ des contraintes résiduelles calculé. 


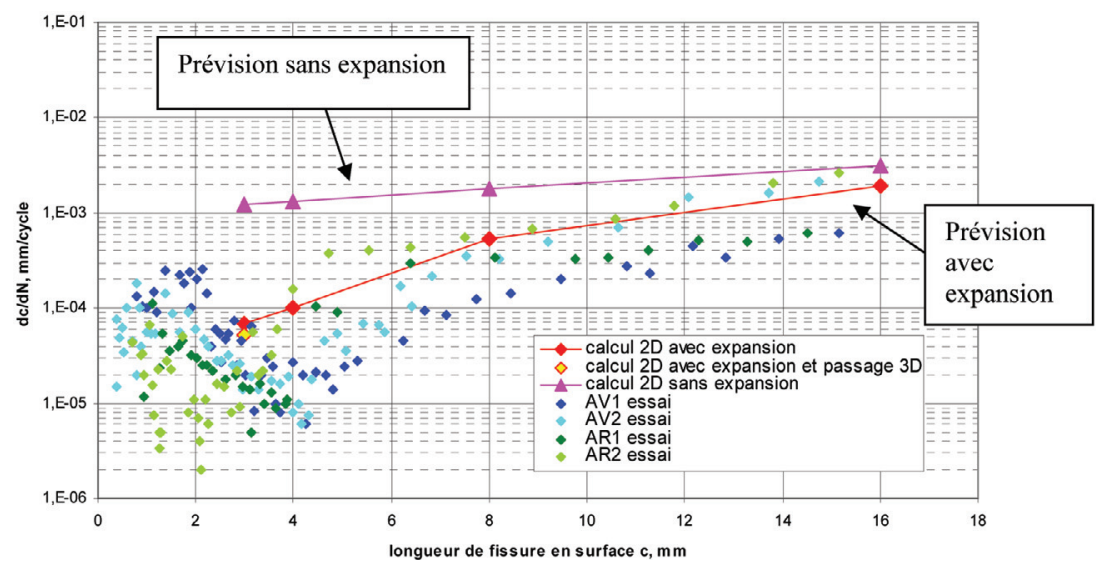

Fig. 20. Cas d'expansion habitée, alliage 2024. Vitesse de fissuration en fonction de la longueur de fissure en surface : résultats d'essais et calculs prévisionnels. Calculs avec défauts traversants pour $c>3 \mathrm{~mm}$ : sans expansion (étape 3a, points avec l'étiquette «calcul 2D sans expansion »), avec expansion (étape 3a, points avec l'étiquette «calcul 2D avec expansion »). Calculs avec quatre défauts coins pour $c=3 \mathrm{~mm}$ (étape $3 \mathrm{~b}$, point avec l'étiquette « calcul 2D avec expansion et passage 3D »).

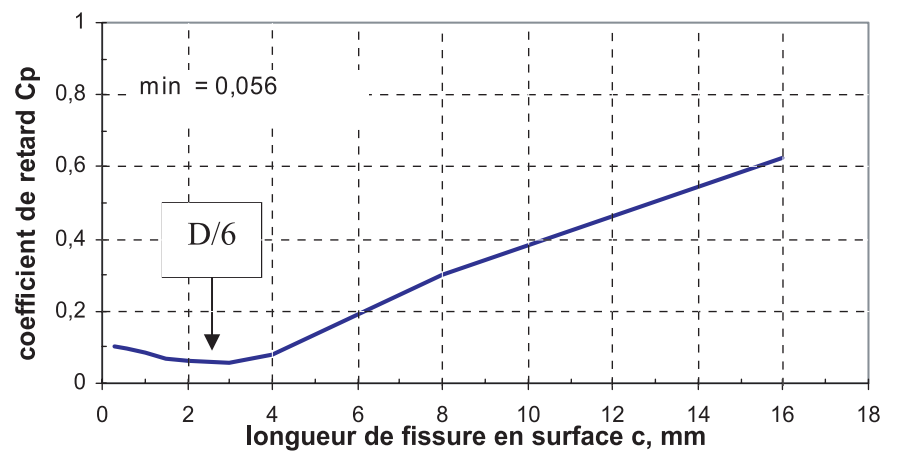

Fig. 21. Cas d'expansion habitée, alliage 2024. Abaque de retard : coefficient de retard $C_{\mathrm{p}}$ en fonction de la longueur de fissure en surface. $D$ est le diamètre de l'alésage.

Tableau 7. Cas d'une expansion habitée. Valeurs de facteurs $K_{w, 2 \mathrm{D}}$, étape 2 , et coefficient de retard $C_{\mathrm{p}}$, étape $3 \mathrm{a}$ de la méthode $\left(S_{\max }=170 \mathrm{MPa}\right)$. Les calculs concernent une fissure traversante, située des deux côtés de l'alésage et de longueur en surface $c$.

\begin{tabular}{ccc}
\hline \multirow{2}{*}{$\begin{array}{c}\text { Longueur } \\
\text { de fissure }\end{array}$} & \multicolumn{2}{c}{ Méthode prévisionnelle } \\
\cline { 2 - 3 }$(c$ & $K_{w, 2 \mathrm{D}}$ & $C_{\mathrm{p}}$ \\
$(\mathrm{mm})$ & $(\mathrm{MPa} \sqrt{m})$ & \\
\hline 0,3 & 11,00 & 0,10 \\
0,6 & 11,98 & 0,098 \\
1 & 12,33 & 0,087 \\
1,5 & 11,90 & 0,069 \\
2 & 11,57 & 0,060 \\
3 & 11,76 & 0,057 \\
4 & 13,25 & 0,078 \\
8 & 22,16 & 0,30 \\
16 & 32,07 & 0,62 \\
\hline
\end{tabular}

Un abaque de retard a été établi (Tab. 7, Fig. 21). L'abaque montre un effet retard important, d'un facteur 17. Le retard maximal est atteint aux environs d'une distance $D / 6$ à partir du bord de l'alésage.

\subsection{Conclusion}

La méthode est vérifiée pour le calcul des facteurs $K$ avec la prise en compte des contraintes résiduelles d'expansion.

\section{Applications [5, 6]}

La méthode offre la possibilité de traiter tous les cas réels d'alésages des jonctions de fuselage en prenant en compte les caractéristiques suivantes : présence d'expansion et/ou d'interférence, niveau du taux de transfert d'effort par le rivet d'une tôle à l'autre.

L'établissement d'abaques prévisionnels de retard à la fissuration, prenant en compte le taux d'interférence et/ou d'expansion pour le calcul des jonctions, permet au bureau d'étude de faire rapidement des calculs simples de propagation, dans lesquels il suffit d'appliquer le coefficient de retard, issu des abaques, aux vitesses calculées sans la prise en compte des contraintes résiduelles.

La figure 22 donne un exemple d'application de la méthode au cas d'un alésage ayant subi une expansion de $4 \%$, habité par un fût de rivet monté avec ou sans interférence de $1 \%$, sous des chargements simulant des taux de transfert de 5, 20 et $50 \%$, au travers de l'alésage, dans une plaque trouée de largeur $50 \mathrm{~mm}$. Les matériaux sont les alliages 2024 et 7075 .

On observe que la vitesse minimale calculée est vingt fois plus faible que dans le cas où il n'y a pas de contraintes résiduelles. Dans cette application, il ressort que pour le 2024 la combinaison la plus efficace est celle procédant à la fois de l'expansion et de l'interférence alors que pour le 7075 l'expansion seule est la plus efficace.

\section{Conclusion}

La démonstration de la tolérance aux dommages des jonctions des structures de fuselage aéronautiques demande de prendre en compte les contraintes résiduelles 
longueur de fissure c, mm

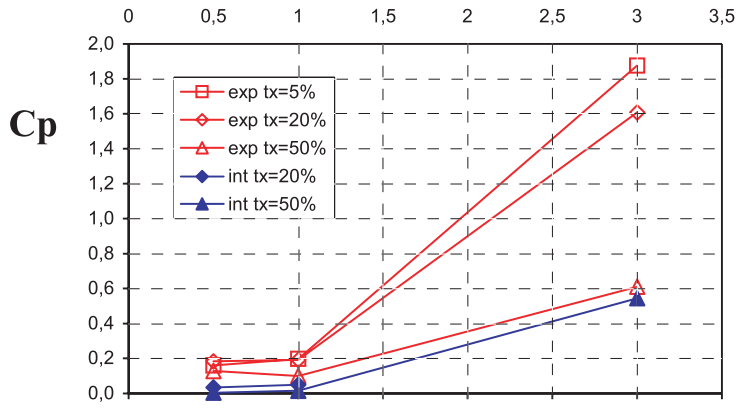

longueur de fissure, $\mathrm{mm}$

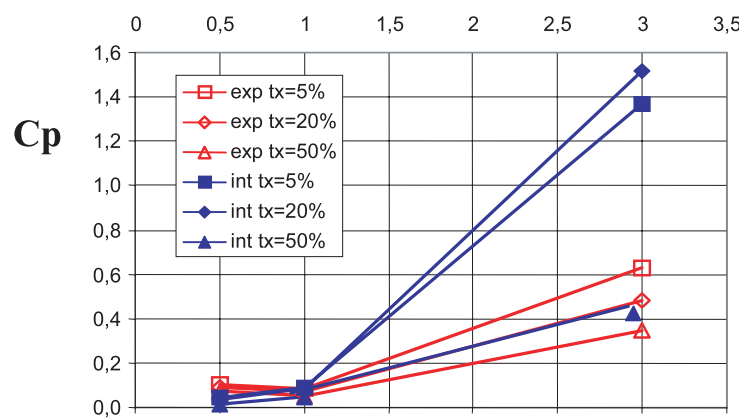

Fig. 22. Abaques prévisionnels de retard, alliages 2024 et 7075, pour un alésage représentatif d'un cas de jonction, avec expansion de $4 \%$ et interférence nulle (étiquette « exp »), avec expansion de $4 \%$ et interférence de $1 \%$ (étiquette « int $»$ ), pour différents taux de transfert tx de 5,20 et $50 \%$, relatifs à des chargements de service nominaux, une épaisseur $B=D / 2$, et un diamètre d'alésage de $D=6,35 \mathrm{~mm}$. Coefficient de retard $C_{\mathrm{p}}$ en fonction de la longueur de fissure $c$.

induites par les procédés de montage des fixations, à savoir l'expansion et l'interférence.

Dans le cadre de la mécanique élastique linéaire de la rupture, une méthode a été présentée pour prédire les vitesses de fissuration, avec la prise en compte de contraintes résiduelles en bord d'alésage. Il s'agit d'une analyse du champ des contraintes en pointe de fissure, obtenu par des calculs élastoplastiques gérant la superposition des contraintes et des déformations induites par le procédé et de l'effort de service, pour calculer la valeur du facteur $K$ nécessaire à la prévision.

Des essais de fissuration par fatigue d'alésages ont été conduits pour les alliages 2024 et 7075 . Trois cas ont été examinés : alésage habité par un rivet monté avec interférence, alésage expansé non habité, alésage expansé habité (effort passant par l'alésage). Les résultats des essais ont montré un effet bénéfique de l'interférence et de l'expansion sur les cinétiques de fissuration, qui se traduit par un retard significatif à la propagation dans les premiers millimètres.

Les résultats expérimentaux ont permis de valider la méthode proposée. Les calculs montrent que l'effet bénéfique est dû à une réduction du facteur d'intensité de contrainte $K$ et de sa variation.

L'application de l'approche à l'établissement d'abaques prévisionnels pour traiter des cas plus complexes rencontrés sur les jonctions rivetées, caractérisés par la prise en compte de combinaisons d'expansion et d'interférence, et des taux de transfert, a été illustrée.

Remerciements. Ces travaux ont pu être menés à bien grâce à la collaboration de plusieurs collègues d'EADS CCR : C. Ithurralde, F. Vinhas, D. Vellay et une stagiaire E. Lucien. Ils ont été financés par AIRBUS-F et pilotés par son représentant D. Duprat.

\section{Références}

[1] K.J. Kang, J.H. Song, Y.Y. Earmme, Fatigue crack growth and closure behaviour through compressive residual stress field, Fatigue Fract. Engng. Mater. Struct. 13(1) (1990) $1-13$

[2] X. Su, M. Gu, M. Yan, A simplified residual stress model for predicting fatigue crack growth behaviour at coldworked fastener holes, Fatigue Fract. Engng. Mater. Struct. 9(1) (1986) 57-64

[3] B. Journet, F. Congourdeau, C. Ithurralde, Fissuration en bord d'alésage, EADS CCR, rapport interne $\mathrm{n}^{\circ} \mathrm{DCR} / \mathrm{M}-$ 64187-97, 1997

[4] B. Journet, E. Lucien, F. Congourdeau, F. Vinhas, C. Ithurralde, D. Duprat, Damage tolerance of cold worked fastener holes, ICAF 2001, Design for Durability in the Digital Age, Proceedings of the 21st symposium of the International Conference on Aeronautical Fatigue, vol. I, Toulouse, France, 27-29 June 2001

[5] B. Journet, F. Congourdeau, C. Ithurralde, Prévision de la fissuration par fatigue des alésages de jonctions rivetées, Actes du Colloque National MECAMAT, Aussois 2003, Assemblages : des matériaux à la structure, 20-24 janvier 2003, pp. 129-136

[6] F. Congourdeau, Perfectionnement des approches petites fissures, calculs avec différents taux de transfert, EADS CCR, rapport interne $\mathrm{n}^{\circ} 2002-24634 / 4-\mathrm{DCR} / \mathrm{SE}$, 2002

[7] B. Journet, C. Ithurralde, F. Congourdeau, Perfectionnement des approches petites fissures : small crack growth behavior, AEROSPATIALE MATRA CCR, rapport interne $\mathrm{n}^{\circ}$ 1999-64634/1-DCR/M, 1999

[8] ESACRACK, code de calcul de facteur $K$ et d'analyse en tolérance aux dommages distribué par l'Agence Spatiale Européenne ESA 